\title{
Looking Outside the Box: Reflecting on Gaze Interaction in Gameplay
}

\author{
Argenis Ramirez Gomez \\ Lancaster University \\ United Kingdom \\ a.ramirezgomez@lancaster.ac.uk
}

\author{
Hans Gellersen \\ Lancaster University \\ United Kingdom \\ h.gellersen@lancaster.ac.uk
}

\begin{abstract}
Gaze interaction in games moved from being a tool for accessibility to be at the core of mass-market game franchises, offering enhanced controller performance and greater immersion. We propose to explore three different popular gazebased interaction mechanics to create novel opportunities in the game design space. We developed Twileyed, a collection of three games that challenge the "common" use of gaze as a pointer to navigate; select; and aim; to pose a challenging new way to play with the eyes. We used the games as data to reflect on the gaze design space. We asked users to play the games to validate them and we observed their experience and strategies. Based on the observations, we discussed through 5 themes the dimensions of gaze interactions and the potential outcomes to create engaging and playful gaze-enabled games. We contribute a position in gaze gameplay design, but also a conversation starter to engage the EyePlay research community.
\end{abstract}

\section{Author Keywords}

Gaze Interaction; Games; Game Design

\section{CCS Concepts}

-Human-centered computing $\rightarrow$ Human computer interaction (HCI);

\section{INTRODUCTION}

Gaze signals interest [58]. Accordingly, we look at the objects we want to interact with, preceding the action [81]. This paradigm has shaped the creation of interaction mechanics focused on gaze pointing, for instance, for selecting targets [34]. With eye trackers becoming affordable and available at a consumer level, gaze interaction has found a place in the gaming industry, promising augmented controllers, improved immersion and enhanced gameplay experience.

In games, by using an eye tracker, players can aim at targets; control the camera movement; tag opponents; or point guns, just by looking at the scene $[43,67,66]$. In esports, and game

Permission to make digital or hard copies of all or part of this work for personal or classroom use is granted without fee provided that copies are not made or distributed for profit or commercial advantage and that copies bear this notice and the full citation on the first page. Copyrights for components of this work owned by others than ACM must be honored. Abstracting with credit is permitted. To copy otherwise, or republish, to post on servers or to redistribute to lists, requires prior specific permission and/or a fee. Request permissions from permissions@acm.org.

CHI PLAY '19, October 22-25, 2019, Barcelona, Spain

(C) 2019 ACM. ISBN 978-1-4503-6688-5/19/10 . \$ $\$ 15.00$

DOI: $10.1145 / 3311350.3347150$ streaming, athletes can share where they are looking so their audience can get a gist of their strategies during the game. Overall, the use of gaze is obvious and provides no challenge. On the contrary, gaze is used to facilitate and augment game performance by moving the action triggered by the controller to the gaze position. In other words, if we seek to shoot an enemy in a gaze-enabled "Tom Clancy's: The Division 2" [19], we need to press a button, and the gun will be automatically aiming at the point where we look. Does this not make the game too easy?

The lack of challenge resides in that the eyes are a natural sensor, and when used as a controller in games at the same time, we might involuntarily trigger unwanted outcomes [70]. This is known to create tension, and thence designers tend to avoid this conflict, adopting gaze in cases where tension is avoided, e.g., looking to select but triggering selection with a button; or slightly moving the camera when looking close to the edges to move the field of view. We challenge this approach and suggest we can deliberately create tension when using gaze in games and analyze the questions and opportunities that arise.

In this work, we want to move beyond the obvious ways of using gaze interactions. We aim to think outside the box and provide an understanding of the different ways of looking at gaze mechanics in games to explore the limits of designing gaze interactions. We designed the game Twileyed with three mini-games that explore commonly used forms of adapting gaze for interaction and used them as a tool for inquiring. In the games, we directly investigate Gaze Selection; Gaze Navigation; and Gaze Aiming, but also include events related to Implicit interaction and Social Gaze mechanics. We describe how we twist these interaction methods in the 3 games with rules and ambiguous (maybe anti-intuitive) gaze mechanics to create tension and open the space for reflection.

The designed games surface as data, and the device to reflect and discuss design challenges, questions, and dimensions of gaze interaction in gameplay. We invited twelve participants to play the games. We observed them playing and collected their insights on the experience. We reflect on the design dimensions of gaze interaction.

Our work contributes to gaze-enabled games interaction research by providing a conversation agenda. Second, we describe three games using gaze interaction paradigms. We used them as data to motivate a discussion and reflection on the 
gaze in games design and its dimensions. Further, based on the presented discussion and design outcomes, future work can engage in continuing the debate on the use of gaze in games.

\section{RELATED WORK}

\section{Gaze in Games}

Gaze was introduced in the game to foster accessibility, by replacing or complimenting the game controllers [30, 33, 31, 57]. Gaze interaction is now set on the target of mainstream game franchises, and more than 140 commercial games have been released to date enabled by eye tracking. Velloso et al. [71] provides a comprehensive survey on gaze mechanics commonly used in games, making available a dictionary of gaze mechanics highlighting the fruitful future of gaze in gameplay. In their work, they summarize the different purposes of gaze mechanics in five categories: Navigation; Aiming \& Shooting; Selection \& Commands; Implicit Interaction; and Visual Effects.

In our work, we draw from gaze Navigation, Selection, and Aiming \& Shooting mechanics to reflect on the limits of gaze interaction, thus using in a secondary place in the game narratives those based on implicit interaction and social gaze (e.g. looks that intimidate; moving the enemies towards the gaze point). Implicit gaze is used, for example, to provide social interaction with avatars [76], or control the camera view [57, $46]$ and rendering [26].

\section{Gaze Selection}

The fundamental principle of gaze interaction is to leverage gaze as a natural pointer and selection mechanism for objects the users align with their eyes. Therefore, an object is selected when it is looked at [15], triggering the object choice and modulating a future event. Once the selection is made, gaze can move away leaving the object selected. This mechanics could be used, for instance, to look at all the objects you want to collect [57]; or to look to select the avatar you want to move.

Gaze selection was made available in games to substitute other controllers, such as mouse and foster accessibility [5]. However, others prefer to use gaze to pre-select and another input device to confirm the selection, e.g., voice; gestures [11]; or touch in an AR application [39]; thus avoiding the Midas Touch problem [70].

Another approach is to use unconscious attention for selection to implement a guessing game [35] in which the engine would "guess" which character the player chose, based on their eye movements and time they look at the selected target. Others used motion matching to select moving targets [77]; or by just looking with dwell time $[12,79]$ to confirm the selection.

In our work, we consider Gaze Selection as the mere act of looking at a character. We want to leverage the Midas touch problem to increase tension in the gameplay and make players trigger unwanted outcomes just by giving attention to the scene. In the game, we use selection to choose the character that the player wants to move.

\section{Gaze Navigation}

Where the eyes look has also been used for the avatar's motion control, by either using a direct mapping between gaze and the avatar position in the screen, for instance, to control the game paddle $[16,74]$; going where you are looking $[57,55]$; or with other input mechanics to confirm the movement [66], such as voice to navigate through tunnels [49], and "jump" between platforms [68].

Other examples used saccadic eye gestures. Saccades are produced when we shift the eyes focus from one point to another (for example, side by side), and has been used to encode the direction of the characters in the space in desktop [32], and mobile games [1]. Overall, gaze navigation is used as a mechanic to "look to go there" [57, 55], or to point at a Cartesian space $[32,48]$, the located areas associated to the different directions on the scene. For example, in GazePilot [48], Nielsen et al. used gaze to steer the direction of the plane in the scene, so if the players were looking at the upper part of the screen, the plane would tilt and point up.

Our work implements the "look to go there" construct, to use gaze to point at the direction the avatar will move towards when motion is triggered.

\section{Gaze Aiming \& Shooting}

Finally, the player's gaze point in games is also used for aiming or direct weapons at a target, and shooting [79, 31, 57]. It is used for automatic firing of firearms and powers [73, 45], or controlled by triggering it with the keyboard.

Generally, gaze is used to support other input modalities; thus the eyes are used to aim at the target whereas mouse [57]; keyboard [61, 31]; touch [51]; hand gestures [73]; or voice [79] are used to confirm the shooting. Others, leveraged gaze aiming to increase the mouse performance in a shooter game [72], by wrapping and swiftly move the mouse cursor towards the gaze point. Moreover, in research games, players can also shoot with gaze only by confirming the shooting with blinks [79]; looking up [30]; or following a moving target in VR [37].

On the other hand, aiming does not always imply shooting. Players can intentionally aim at the game to point with a torch [3, 73]; aiming attention to the opponent to stop them [38]; or firing the power to freeze enemies [45]. However, here, we use multimodal interaction to aim with gaze and trigger the shooting with the keyboard.

\section{Ambiguity, Reflection, and Games}

Ambiguity is the uncertainty and inexactness that obscures the meaning or use of something, inviting to create multiple and rich interpretations. In Human-Computer Interaction (HCI), ambiguity has been seen as a problem, but it is introduced as a resource and an opportunity for design [54].

Gaver et al. [23] described the advantages of enabling designers to go beyond the limits of their technologies by devising ambiguity in their applications. In their view, ambiguity is a powerful design tool that provides a framework to use inaccurate sensors and inexact mappings to create engaging and thought-provoking interactive applications that encourage 

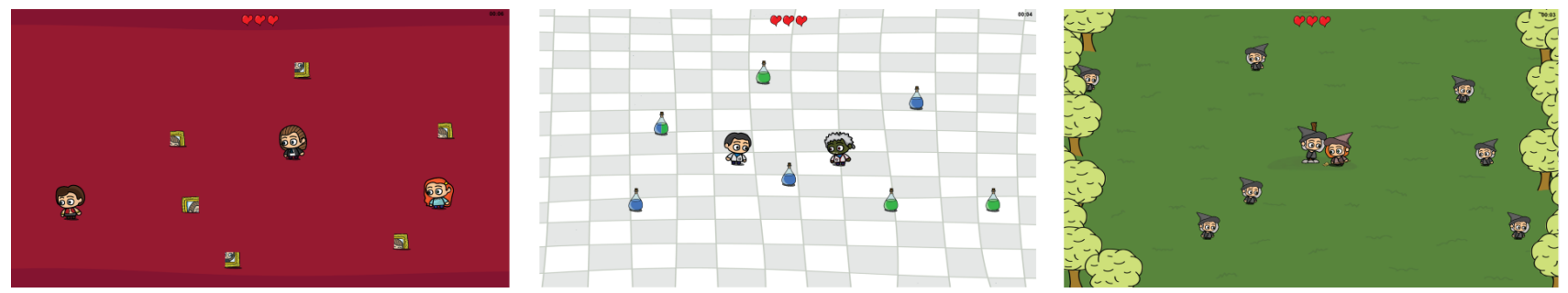

Figure 1: Twileyed's three games' scenes. (Left) Dorian's Pictures. (Center) Jekyll and Hyde. (Right) The Witches.

users to make their interpretations. Moreover, they claim ambiguity allows designers to give perspective and suggest issues to be considered rather than imposing solutions.

In our work, we use ambiguity in the design of the gaze mechanics in the games to introduce tension. Besides, it allows us to stretch the limitations of eye tracking in games giving the opportunity to renounce to propose solutions or answers but to question and raise themes for discussion, opening the space for reflection.

Accordingly, reflection is a necessary component of learning [8] and has been broadly used within the HCI research community [21, 6]. Reflective Design is concerned with how we considerate and think about a product according to subjective factors, and it is essential during the design process [56].

In games, players usually reflect on the gaming experience in online forums and game communities; thus games have been used to encourage reflection in research [24], with a focus on the player perspective $[36,42,50]$, but also used by designers to reflect on the game design process [20,29]. Harteveld et al. [25] reflected in their work on the tension of game design, analyzing, for example, the dilemmas on the design process.

In our work, we reflect on the player experience from the gaze-enabled games' designer perspective rather than asking players to reflect on the game itself. We propose to focus on observations of the users playing the games to question gaze interactions, and therefore, to learn and discuss the design opportunities that ambiguity raised.

\section{TWILEYED}

We developed the game Twileyed containing three mini-games that challenge the use of gaze interaction (see Figure 1). We represent each game by characters, mostly from fiction, representing "duality" to implement the ambiguity in the different gaze mechanics. The games aim is to complete a collection task with challenging interaction. Gaze interaction is used to modulate navigation by selecting characters to move; aiming direction; or shooting with gaze for protection.

However, we designed each game by thinking about how to increase the tension and add ambiguity to the gaze controls that, without the challenge, would more easily be performed. Players need to learn how to use the interaction mechanic and adapt their strategy to overcome each game challenge. We aimed to provide three examples of games that include gaze interaction to explore the potential applications of gaze in games.
We chose to use multimodal interaction for the avatar control to solve the task combining Gaze and the Keyboard. The Arrow/WASD keys are used to move the avatar in the games (either up, down, left or right), whereas the space key can be used to trigger events; e.g., an attack. Using this dual interaction allows the concept to split between the fundamental controls provided by the keyboard, and the modulation of such with the use of gaze. In the presented games, both inputs for interaction are dependent on each other and co-exist in the game environment. Therefore, the player cannot overcome the game challenge if one of them is not available.

Moreover, playing with "tension" requires the definition of game rules that create ambiguity of context [23] of the gaze mechanic to disrupt the interaction with incompatible uses; add functions to break the gaze dynamic; and modify the expected functionality.

\section{Gaze Selection - Dorian's Pictures}

In the first game, gaze is used for Selection. To move a character, players need to look at them to select them. However, the ambiguity in the interaction context is articulated by setting opposed interaction dynamics for selection.
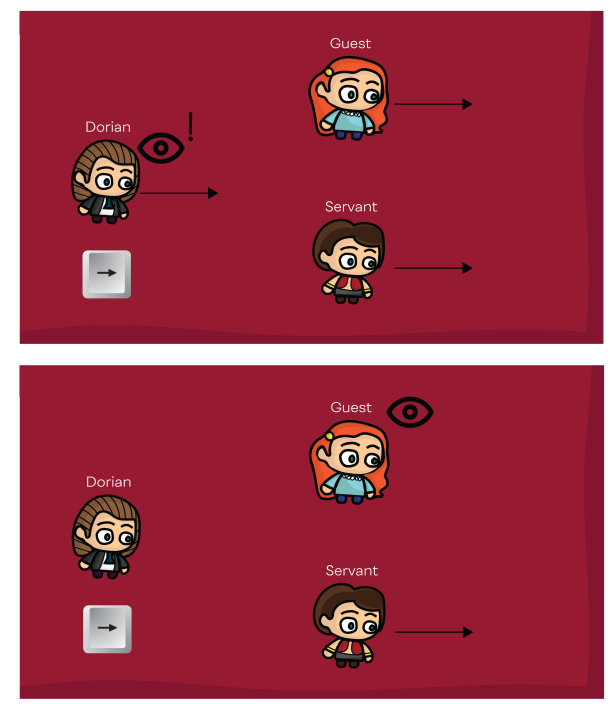

Figure 2: Gaze Selection in Dorian's game. (Top) Dorian needs to be selected to move. (Bottom) Selecting other characters disables their motion. 
The game story is based on the literary character of Dorian from "The Picture of Dorian Gray" by Oscar Wilde [80]. Although Dorian is immortal, he could be killed if he is in front of his picture.

In the game, the player controls Dorian, who is at a party populated by Guests, Servants and Assassins. The aim of the game is to move Dorian and collect the pieces of his picture, before anyone else collects them (see Figure 1, left). In the meantime, the Assassins will attempt to kill Dorian. Servants and Guests can stop the Assassins by getting in their way. When that happens, Guests will be killed by the assassin, whereas Servants will remain in the game. Once the Assassins are intercepted, they escape and wait until the next attack.

Dorian is very narcissistic and requires all the attention to move. Moreover, Dorian is the host of the party, and when he moves, everybody else will move to try to win his favor. If $D o-$ rian looks at the Servants or Guests, they will feel intimidated (implicit "social" gaze) and stop moving.

The game challenges the players in forcing them to select with gaze Dorian to make him move, and risking the rest of the characters collecting a piece of his picture (Figure 2, top). Further, if the player selects at any of the other characters, only the unselected characters will move (Figure 2, bottom). This can become handy when avoiding the Assassin's attacks.

\section{Gaze Navigation - Jekyll and Hyde}

In the second game, gaze is used for Navigation. Gaze is used to point at the direction of the movement. However, the tension in the interaction context is articulated by biasing the gaze mapping.

The game story is based on the story of Dr Jekyll and Mr. Hyde from "Strange Case of Dr. Jekyll and Mr. Hyde" by Robert Louis Stevenson [59]. In the story, Dr. Jekyll would transform into his alter ego $\mathrm{Mr} \mathrm{Hyde}$ by drinking a special serum.

In the game, Dr Jekyll managed to separate himself from Mr Hyde and both need to drink a serum to keep it that way. However, the solution has a side effect, and $M r$ Hyde will only move when Dr Jekyll moves. The aim of the game is for each to collect their appropriate serum bottles, Jekyll the blue ones, and Hyde the green ones. However, they cannot collect each others' bottles. Moreover, if they touch each other, they will merge into one single person again, until they gather together the red serum to separate again. Nevertheless, when both merged, they can collect the bottles with mixed serum, blue and green (See Figure 1, center).

The player can control Dr Jekyll with the Keyboard, and the side effect from the separation serum makes Hyde move only when Jekyll moves. However, the experiment made them connected and Hyde will move "through Jekyll's eyes". This means that Hyde will move when Jekyll moves towards the direction the second is looking at (see Figure 3). As a result, Jekyll turns into the center of a Cartesian space for the movement of Hyde (in other words, a joystick controlled by gaze using Jekyll as the center, position [0,0]). For example, if Jekyll goes to the right but is looking to the top-left, Hyde will move towards the top-left. Further, if both characters merge

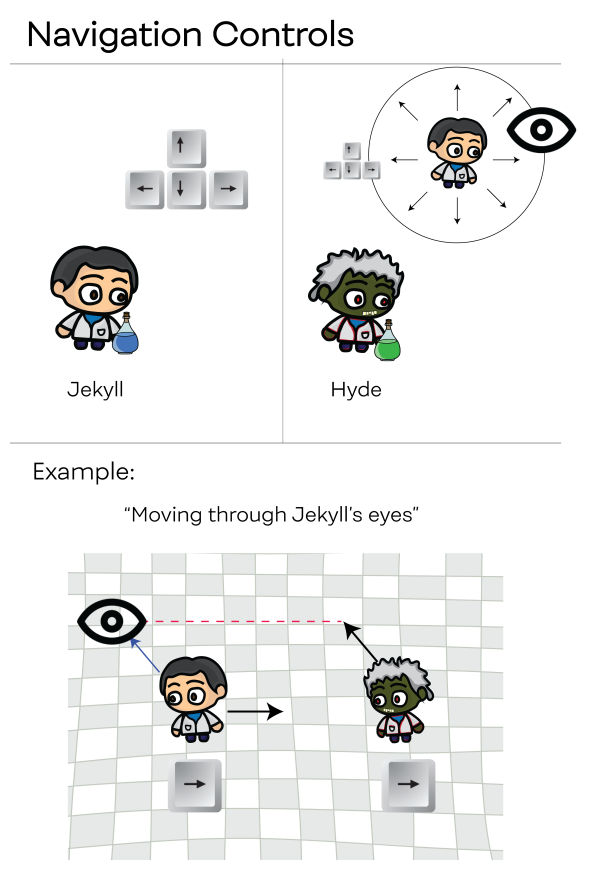

Figure 3: Gaze Navigation biased mechanic in Jekyll and Hyde's game.

and become a single character, gaze navigation has no bias, and the avatar will move towards the gaze point when the keys are pressed.

The game challenges the players to understand and work out the navigation mapping to move both characters at the same time without collecting the wrong serum. The gaze mapping is anti-intuitive, and some times the player might need to look towards the opposite direction the main character is moving.

\section{Gaze Aiming - The Witches}

In the last game, gaze is used for Aiming \& Shooting. The main character can shoot, and players need to look to where they want to point the attack. However, the tension in the interaction context is articulated by giving Gaze Awareness to the game opponents, thus spoiling the player strategy.

The game story is based on both historical and fictional figures of the Witches during the Witches Trials. We set the game during a witch hunt in which a witch is going to be burned at stake.

In the game, the player controls a Witch that needs to recruit other Witches in the forest to help the captive fellow Witch to escape (see Figure 1, right). Witches need to be collected one by one and carried to the imprisoned witch. During the task, there are Villagers hunting for them and will kill them at contact; some wander around the scene, and others led by the Priests will try to burn the prisoner witch.

The player can cast water spells, aimed by gaze, to extinct the flame keepers fire and make them run away for more (Figure 4, top). Villagers only need one hit to get scared but the evil Priests keep a second flame with them and will 

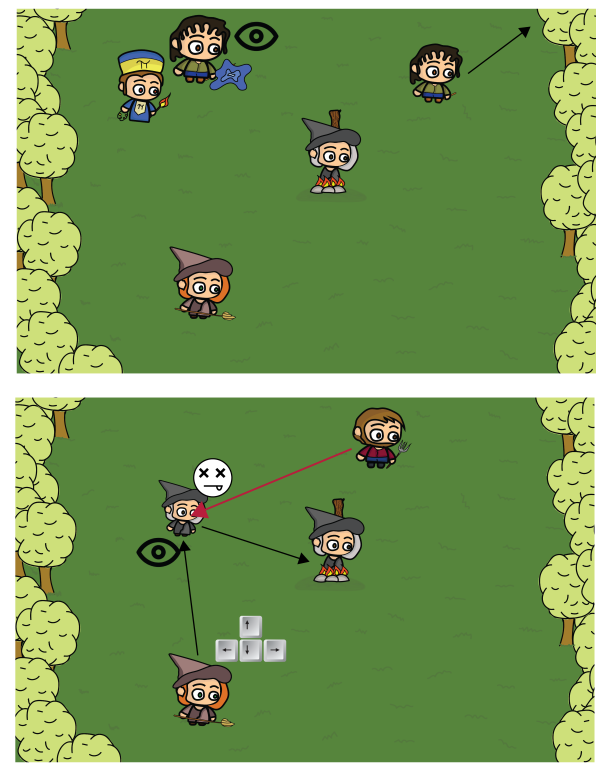

Figure 4: Gaze Aiming uses in The Witches' game. (Top) To shoot at enemies with the space key. (Bottom) Enemy going towards the gaze point of interest.

need to be hit twice. However, magic always comes with a price. One of the Villagers can detect where magic is about to happen and will move towards the point where the main Witch (player) is looking at (Figure 4, bottom). Further, if the Smart Villager encounters other Villagers or Priests, they will join him forming a big horde of people in search of the source of magic.

The game challenges the players to look to sense the scene while solving the task and aim at enemies to shoot, but also avoid to give up their strategy (Figure 4). When players want to collect a witch, they would naturally look at their target (implicit gaze). However, this will make the enemy go towards the targeted witch and kill her. Similarly, when attacking the opponents, looking at them for too long can make the enemies group.

\section{Implementation}

Twileyed was developed using Unity Game Engine in 2D, custom graphics and creative commons sounds. The game requires the use of a keyboard for game control, an eye-tracker and uses Tobii Gaming SDK for Unity to enable gaze interaction. The calibration of the eye-tracker is required before playing the game. The 3 games have infinite levels, once the goal is achieved, the level goes up, placing another set of collectible objects in new randomized locations.

\section{GAMEPLAY EVALUATION}

We invited 12 users to individually test the concept introduced in each of the games in Twileyed. We used a TobiiEyeX eyetracker under a 27" monitor (Resolution: 1920x1080; Aspect Ratio: $16: 9$ ) at $40 \mathrm{~cm}$ from the user and a keyboard to play with the games.
In the session, we gathered participants demographics data, including close-ended questions about the frequency they played video games, type and mostly used device platform. Then, we asked participants to play each game freely for 15 minutes, but they could play longer if they wished to do so. At the start of each gameplay, we explained the instructions and story of the game and calibrated the eye tracker by using Tobii native application. After playing each game, participants were required to answer their level of agreement with three statements related to the increased tension of the interaction, and evaluating the level of challenge of the game; perceived competence; and willingness to play again (Q1: "I felt challenged"; Q2: "I felt I did well"; Q3: "I would like to play again"). Each statement was rated on a 5-point Likert scale ( $1=$ "Not at all"; $2=$ "Slightly"; 3 = "Moderately"; 4 = "Fairly"; 5 = "Extremely").

During the session, we observed the participant playing and took notes of their game strategies, actions, and out-loud comments. Each session duration was around 1 hour.

Twelve volunteer participants played the three games. Players were aged 21-35, with a mean of $27 \pm 4$ years old. From the seven men and five women who volunteered, six wore glasses, six have had previous experience with eye tracking, and four were used to play video games regularly (between once a week and almost every day).

The aim to evaluate the player experience was to analyze the effect of the increased tension while using the gaze interaction mechanic and verify we provided well-designed gameplay, both challenging but enjoyable enough to generate interest to play again.

Figure 5 show the results from the evaluated statements, indicating that the three games were perceived as fairly to extremely challenging; most players did not (to moderate) feel competent at playing them, but overall seemed moderate to extremely keen to play again. These results suggest the gaze mechanics posed a big challenge but were able to engage the users in the gameplay. Although the player experience results give an insight on how the players perceived the experience in terms of difficulty of the games, competence and enjoyment, the data does not provide a clear conclusion to be further discussed in the following sections.

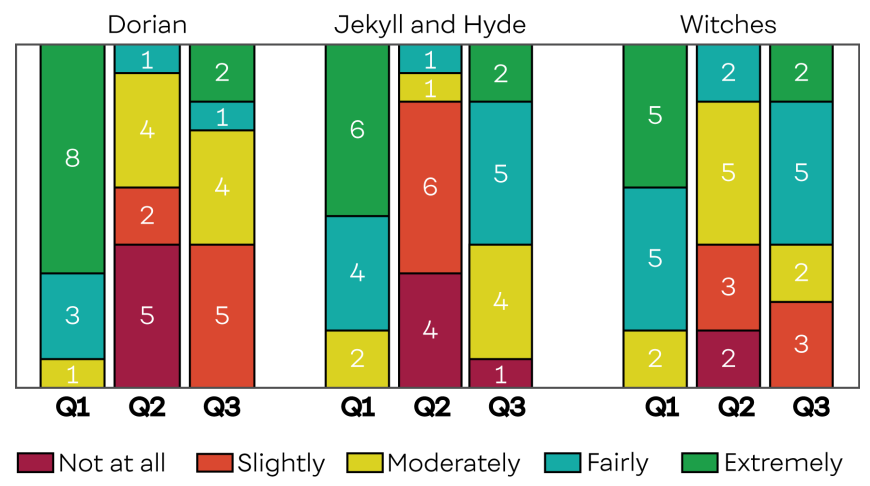

Figure 5: Player Experience Results for each game 
Table 1: Final codes' set for the thematic analysis.

\begin{tabular}{c|l|c} 
Theme & \multicolumn{1}{|c|}{ Code Set Name } & Data Units \\
\hline 1 & Trouble handling game events & 35 \\
\hline \multirow{2}{*}{2} & Visual attention issues during the game & 21 \\
\cline { 2 - 3 } & About looking away and not looking & 13 \\
\hline \multirow{2}{*}{3} & Learning to play and managing skills to succeed & 16 \\
\cline { 2 - 3 } & Tactics and strategy development & 11 \\
\hline 4 & Interaction understanding, meaning and perception & 14 \\
\hline 5 & Changing the game rules, twisting the game dynamics & 35 \\
\hline All & The game experience: challenge, enjoyment and tension & 35 \\
\hline \multirow{2}{*}{ None } & Gameplay description & 21 \\
\cline { 2 - 3 } & Other &
\end{tabular}

\section{Gameplay Analysis}

We performed a thematic analysis of our gameplay observation notes. The format of the data units was of varying lengths (words, short sentences, and long paragraphs). They ranged from a literal description of the events narrating the order the players followed to solve the game tasks; their reaction to the game events; the observer comments; summaries of the participants' observed strategies; and participants' out-loud quotes and opinion extracts. There were a total of 216 units. We examined the data to develop an initial set of 19 initial codes and grouped the instances in these codes. We refined them, discarding those with little repetition, and discussed within the research team the relevance of the rest, resulting in the creation of 10 themed set of codes based on their common patterns (see Table 1). Lastly, we developed a final set of 5 themes based on questions raised by each summarized set regarding the games' challenges, presented interaction concepts, and the mechanic outcomes.

\section{REFLECTING ON GAZE INTERACTIONS}

In this section, we present five themes unpacking questions on the player experience and interaction mechanics based on the observations. The themes might provide implications and a set of multiple dimensions for researchers to analyze gaze interactions in games. For each topic, we reflect and discuss how they resulted from the game, the questions that emerged and design implications aimed towards guiding the future directions of gaze-enabled games.

\section{Theme 1: Attention to the Interaction}

The games in Twileyed are challenging by design. The games aimed to create tension with gaze interaction mechanics. However, we observed how overcoming the interaction challenge inhibited the completion of the games' tasks.

In a nutshell, the three games had a collection task set as the main objective. Players needed to collect all the available objects in the game scene. However, at the same time, there could be other simultaneous events interrupting the primary objective. In Dorian's game, the player faces a Survival task when the Assassin tries to kill the main character, and a Prevention task, to avoid that the other characters collect the objects in the scene. In Jekyll's game, the player needs to prevent the characters from picking up the other serums. Finally, in the Witches' game, attacks from the villagers carrying flames must be survived, while stopping the other opponents from killing the witches.
This simultaneous juggling of events is not a significant challenge, because games usually integrate different parallel tasks during gameplay. For example, in an adventure game such as "The Shadow of the Tomb Raider" [43], Lara Croft might need to retrieve an artifact, but in the way, she might be attacked by enemies and need to deal with them first, interrupting the main task. However, this handling of two simultaneous tasks became a challenge in our games. The player needed to prioritize which tasks comes first while remembering the rules of gaze interaction in the game.

In Dorian's game, we observed two strategies being adopted. The first was to focus on the selection paradigm, forgetting about the Assassins' attack, and thus failing when that happened. The second was to move away from the other characters and collect the objects without having to worry about them. Consequently, when the Assassin attacked, they had to rush, tried to move them by looking at them (as they did with Dorian), and thus not moving at all and failing.

Does this mean we can either be attentive to the interaction or the task at the same time? One can say, the different interactions were anti-intuitive; you cannot require to select one character by gaze to move, but avoid looking at the other to do the same. In general, this might suggest gaze interaction must avoid using tension in interaction, or that when using tension only one task should be presented.

One might disagree with that. If we think about playing Flappy Bird [47], the aim of the game is to make the bird flap upwards to navigate through pipes. When playing for the first time, nobody expects the bird to be affected by gravity and fall if we do not tap the phone screen immediately. The game could be quite addictive while creating tension. Now, let's re-imagine it. If in a gaze interaction version of Flappy Bird we need to (anti-intuitively) look up to go down, and down to go up when we tap, would the simple fact that we can be bad at it makes us want to play again and challenge ourselves? In the same way, the players in Twileyed were frustrated by the challenging interaction but wanted to keep playing and push themselves to try again.

If we look at the opposite side of tension and think about games that use gaze to support interaction, for example, in an action game [19] we need to decide whether one of the characters is good or bad to attack them. In the game, by looking we can tag them and know the information beforehand. Does this remove all challenge in the gameplay? 
Sometimes what is not intuitive and natural can be fun and engaging. For example, in Virus Hunt [73] players could not look at the screen to remove the virus, or it would reproduce, creating tension with gaze interaction in a playful and fun way. In Invisible Eni [18], the user needs to close their eyes to make the character invisible and protect her from enemies. Not looking as an interaction with a technology that fosters looking seems thought-provoking. Therefore, we ask whether as gaze-enabled games designers, do we want to create engaging experiences even though they are challenging and affect the players' attention to both the game and the interaction? We propose to think and explore challenging gaze interactions with tension when designing games.

\section{Theme 2: Visual Dilemmas}

Similar to the attention to the game events, the players faced the challenge of dealing with attention dilemmas. When we look, we are limited to focus our visual attention to one object at a time. In games, this requires peripheral awareness to detect events that happen where we are not looking.

In Twileyed the tension in interaction inevitably builds on creating an attention dilemma. When moving Hyde "through Jekyll's eyes", we are making players look away from the character they are modulating motion with gaze, and thus keeping him in the visual periphery. Besides, when players look at Dorian to move, that cannot look at the other characters and the other way round. They will need to invigilate the other character always on their periphery. Lastly, in the witches games, if the player looks at a witch while moving the main character to collect her, it would draw the attention of the villager; Then, to prevent that they needed to perform the action looking away, and therefore without explicitly looking.

Some games build on attention dilemmas. In Shynosaurs [75] the players need to stop looking at the main task, to stare at the enemies to scare them away. However, while in Shynosaurs the attention dilemma is based on the occurrence of two simultaneous events and the limitations of vision to look at two things at the same time; in a way, in our games we force the attention dilemma into the game dynamic by adding consequences for looking for interaction, and this makes players rely on peripheral vision.

During the playing sessions, two participants referred to having to balance how much they looked away from the screen and use their visual periphery (Participant 1 and Participant 5, P1 and P5). This suggests, their visual attention was in peripheral vision, but relying on peripheral vision means they will see objects with poor acuity, blurry, and shape-distorted [4, 78]. However, peripheral perception is essential in tasks like object detection [53]. Therefore, how could gaze interaction benefit from the capabilities of peripheral vision?

In games, this capability has been used to display effects where we are not looking, in our periphery, to create a scary immersive effects [28]. Moreover, the term peripheral interaction is used to describe to those interactions performed to something that is not in the focus of your attention, and it is referred to be short [2]. Does this mean that by relying on the visual periphery, we are making the focus of the gaze interaction short?
How can we leverage the abilities that we have in vision when the game does not allow us to look?

Peripheral awareness exists in games, but one might think it is passive and it is only used to shift attention. For example, in Shynosaurs [75], the player needs to be aware that enemies are approaching to change their visual focus to deal with them. With Twileyed we created a game that makes players necessitate to rely on peripheral vision and made focal interaction brief. In the witches' game, the player is required to look away, where there is no game action until they have to scare an opponent away. Is, therefore, brief gaze interaction a new paradigm to consider? To what extent is peripheral vision an opportunity or a burden in interaction?

Virus Hunt [73] and SuperVision [52] are two gaze-enabled research games that explore the use of peripheral vision in play. They challenge the players to leverage their perception abilities in the periphery of their vision to sort and manipulate objects in the game by using gaze aversion mechanics to penalize the players when they look. However, there are not enough examples to generalize how designers could use peripheral vision to create new gaze interaction paradigms beyond forbidding the player to look, and what new opportunities could be created for gaze-enabled games.

\section{Theme 3: Anti-intuitive Gaze Interactions}

We reflected on the interaction dynamics created based on the feedback players gave on the game challenges. Overall, adding tension and anti-intuitiveness to the gaze interactions made the games difficult but engaging, and they encouraged players to play more.

As discussed in previous themes, gaze can have creative uses that make the interaction challenging. In Twileyed, we used changes or consequences to the interaction mechanics by using gaze. During design, we decided that tension could be applied to add handicaps affecting how players use Gaze Pointing and pushes them to think about how to use the interaction mechanic in a way that might not be immediately intuitive. We made the gaze interactions inherently difficult to make players experience complex character control by setting rules based on:

\section{Imposition:}

When gaze is used for selection, the player can be forced or restricted to look at a specific element only. For example, in Dorian's game, the player must either look at the avatar or avoid to look at them to make them move. Therefore, although several elements might be available for selection, the rule limits it to one at a time.

\section{Biased-Mapping:}

It is applied when gaze pointing is used to direct the avatar's motion. The logical relationship between the player's gaze position and the avatar's position in the game scene can be skewed or modified. For Hyde's movement, we set the direction by gaze but translating its origin to Jekyll's position. Other examples could repel the point where you look and move away 
from it; move towards the opposite side by mirroring directions, or moving the Cartesian space away from the avatar and introducing drifts on the mapping.

\section{Spoiling:}

When the player uses gaze as a pointer for interaction, the players are giving away their strategy. Similarly, the game is aware of where the user is aiming with gaze and can use this information against them (e.g., placing enemies in their way (Witches); or even moving collectibles away from where you look).

Looking back, some players reported to "need time to develop the logic" (P11) or thought the games were "giving room to get more skilled if they played more" (P2, P6, P12). They described while playing the mechanics to be "anti-intuitive" (P3) but "interesting" (P4), making them "think a strategy" (P3, P5) to perform something that a priori "does not look that difficult" (P8). Could therefore tension and anti-intuitiveness be useful to design new playful gaze interactions?

We can see tension and anti-intuitiveness as a side effect, or the secondary consequence, that happens by using gaze. Interaction with the eyes does usually help the player in the gameplay. However, what if gaze interaction had a price? Designers can add tension, the same way a reward is given. Maybe in a game using gaze interaction, the avatar can eat a berry that makes them intoxicated, and wherever they look is not where they are going when using gaze for navigation. Others, like in The Royal Corgi [76], might want to impose that you look at the character while you act, as if avoiding to get caught and checking the other character is not looking at you.

We are not implying that gaze interaction needs to be difficult by design, but why would it not include side effects? The use of inaccuracies could serve as a source of design. We want to challenge the game design community to think about the possibilities that an inaccurate interaction could bring to the creation of creative games.

\section{Theme 4: The role of Metaphors}

Gaze interaction is often presented as a feature that augments the controller [22], for example, to extended view; clean UI; interaction at gaze; dynamic light; center at gaze; fire at gaze; among others. However, when you look at creative ways of using gaze, the interaction is described in a narrative and somewhat poetic way, introducing looks that intimidate [75], others could freeze [45], or even charm [13]. In Watch Dogs 2 [65], for instance, one can hack someone by just looking.

During gameplay, players referred to the use of gaze in the games, describing it as something "necessary" (P5); "having a point" (P3, P5); and even talking out loud about the interaction to say they needed to "make eye contact" (P11). This made us think about the meaning of gaze, and how storytelling and metaphors could create novel interactions with gaze.

In the game stories, we set a narrative to use the different interaction mechanics and give a context. Our initial idea was to influence how players interact with the game contextualizing it in the embedded story. However, it was not our intention to give it meaning as such, but we used metaphors as inspiration.
We can analyze one of the metaphors used as "Psychical". They relate to phenomena that are inexplicable by natural laws and affect the characters' behavior. For gaze interaction, it could be aiming magic with gaze but also leaving a trail that can be followed (Witches). We can also consider a "Symbiosis" metaphor, to refer to two characters that can be connected making the actions of one affect the other, and there is a dualism between their identities ("Moving through Jekyll's eyes").

On the other hand, we also used "Social" metaphors. They relate to society, character's hierarchy, personality, and social behavior. For instance, the narcissistic behaviour of Dorian, meaning a character can require the player's attention to move. Other characters can get shy when the players look at them and do not move. The metaphors could also affect the "Physicality" of the character (physical body or representation). For instance, to create a reaction from actions, meaning that the action from one character will affect others.

We see the potential of creating compelling interaction by giving them a meaning, especially when they have a social meaning. The eyes are part of social communication, and there are examples of social gaze in games [76]. In The Royal Corgi players need to be careful where they attend with gaze while conversing with the different characters, this could modulate the story by translating interest into social behaviors like being shy, daring, or even rude. If the eyes are used in the real work to show attention, why not use it in a game?

In mass-market games, gaze attention to the scene has not been explored much. In Dying Light [62], zombies would notice the players if they look at them. Similarly, in Assassins, Creed [66], passers-by will wave at the player's avatar when looked at. Considering meaningful gaze interactions as those which make sense to the user and are not arbitrary. What could designers do to introduce more meaningful uses of gaze in games for playful interactions?

If we follow on the use of metaphors, gaze signals attention, and the lack of it would accordingly, show disinterest. Could this social gaze metaphor fit in the game narrative? For example, in Tomb Raider [43], we can decide to talk to an NPC (Non-Player Character) during the game. You can move away from the NPCs, and they will continue talking. We could think of a new interaction like in The Royal Corgi, and make conversations evolve by gaze attention. However, what if the NPCs could have different personalities, and one, like Dorian, needs all eyes on him. Would the lack of eye contact make the character stop the conversation and angry move away?

Whether this would be compelling for players, is yet to be seen, however, as designers we think there is potential on creating such grounded in the narrative interactions.

\section{Theme 5: Gaze's Identity and Control}

During the Witches' game gameplay, players pointed out how much they were enjoying being able to control the enemies and use them as their "minions" (P3, P4, P8, P11) to protect the captive witch. It was not what we intended when designing the game, but what happened is that the enemy would move towards the player's gaze point, and when colliding with other 
opponents, they would join him forming a group. This was first envisioned as a burden, the group of enemies would increase, making them bigger and harder to avoid they caught a witch and kill her. Ironically, players turned this issue around into their favor, considering that in fact, they were controlling the enemy with gaze. However, gaze was supposed to control the powers of the witch. Therefore, who is gaze representing? What is the identity of gaze?

We refer to the player gaze identity to what or which game element the user's eyes are related to and therefore it is used for interaction control. Traditionally in gaze interaction, the eyes could represent or adopt either the main character gaze or the users themselves. What this means is that where the user looks can be considered, correspondingly, where the character is looking, or where the user is looking. Technically it is the same, but contextually in the game narrative, it is different. In a way, we could say that identity could be an iteration on the meaning of using gaze; however, we believe it is a topic for discussion alone.

In Twileyed, accidentally, we assigned double identities to gaze. When playing Dorian's game, looking at Dorian is the user's attention, but when gaze selects the other characters, it is Dorian who looks at them and intimidates them. Does this design support gaze identity being the Player and the Avatar?

Jekyll and Hyde were presented as the two characters of the game. One can say that both are player's avatars, but also because the player can only move Jekyll and Hyde acts accordingly, Hyde could also be the avatar's Companion in the game. Then, if Hyde will move towards where Jekyll is looking, is gaze's identity Jekyll, Hyde, or both? It could be Jekyll by meaning and through the use of metaphors, but it could also be Hyde alone because it is he who gaze controls with a biased mapping. Therefore, does this design support the concept of double identity? We could argue that it only does by definition of the metaphor, but if both characters merge and gaze pointing is used for navigation of the single avatar, is both of them then gaze's identity?

Finally, in the Witches' game we allowed the control by gaze of the avatar powers and the enemy at the same time. Gaze belongs then to the Avatar and the NPC (Enemy) correspondingly. But what is "Identity"?

In games research, identity refers to the user as the player, and the player as "gamer" [14]. Identity can also be a playercharacter relationship within the game [44], meaning that the player identity in the game is the Avatar. Generally, Avatars in games allow players to project their identity into the virtual world [17]. When customized, avatars can stimulate identification [7], but also a playful exploration of oneself identity [69], or experience gender identity swaps [27].

Moreover, identifying with an avatar, usually, through game controllers [44], can have a positive outcome on player experience and enjoyment [63]. For example, Carter et al. explored the effects of identity dissonance in games using voice interaction [9]. However, they differentiate between 4 identities, that may overlay and inform each other during gameplay [10]. They put forward: the user (the "real" person playing the game); the player (the social identity); the character (the identity within the game narrative); and the avatar (the character's virtual embodiment). In their example using voice interaction, through voice control in games they could overlay the player and character identities.

Therefore, is gaze as a controller a bridge to facilitate identification with the avatar? If so, we could consider that in our game examples there was an overlap between the player and the character identity, and thus gaze's identity that belongs to the user is mapped into the other two. Nevertheless, what is the rationale in a first camera perspective game? Is gaze identity mapped into the character, the avatar, or is it the player in an immersive context? The answer could have multiple interpretations.

Overall, the gaze interaction used allowed to introduce the possibility of the user's gaze to assume a dual identity during the games (Avatar and Player, Avatar and NPC), but they also enabled to control characters (e.g., NPCs) that were not possible to do so before, such as companions or enemies.

We can find a similar dynamic in Brothers: A Tale of Two Sons [60], a game that introduces two characters that need to be controlled at the same time with the two halves of the game controller. When looking at gaze interaction, Far Cry 5 [67] introduces the feature "Guns for hire" which is used to show your allies where to move by pointing with gaze. This last short interaction faces the same dilemma for gaze's identity attribution, and overlays the character's avatar and the NPCs'. We could argue that in this case, like in spy-movies, the main character could signal with the eyes where to go, and thus gaze pointing belongs to them alone. However, by pointing with your eyes, you can make the others look at the same position. Then, are they both gaze's identities one by narrative definition and the other by control?

Other research works related to gaze and identity have a focus on social presence. For instance, when the player's gaze point visualization and the player's character (avatar) are present in the scene, the users might not be sure if in the game they are the character or the gaze visualization [40]. Similarly, when a second user is present, gaze identity could not necessarily be related to the primary user and player but can represent this second spectator, allowing them to have a more active role within the game rather than being mere audience [41]. These examples investigated how different gaze point visualizations during the game may create an ambiguity of the player identity and perceived presence in the game. They relate to a nondiegetic identity of the game that is outside the game story and aligned with conversations about gameplay and control. Here, we discuss the diegetic identity of the players' gaze within the gameplay narrative, and we propose to investigate "identity" based on what does it mean for the player to interact with gaze in the game.

On the whole, we encourage designers to think about gaze identity to create rich gaze interactions based on relationships of attention to the game scene and explore such a broad topic such as Identity. Moreover, we see the potential to study player-avatar identity when using gaze interaction. 


\section{DISCUSSION}

In this paper, we presented a conversation starter. We discussed the questions and outcomes constructed upon reflection on our game design. The reader can consider this contribution as an opinion, a provocation, or an invitation to think about topics that are not usually discussed within the gaze-in-games community.

Research contributions on gaze interaction for gameplay are populated with user studies to evaluate a novel interaction mechanic and describe their implications for design. To find conversations on the broader opportunities to design with gaze, we need to refer to surveys. In Velloso et al. work [71], they provide a general view of EyePlay applications, coined term to talk about the playful experiences that take input from the eyes [64]. Their approach is to present games that use gaze for interaction with a technical perspective based on eye movements; input types (discrete and continuous); outcomes of gaze mechanics; and sensor shortcomings. In their survey they describe the future of EyePlay and its research agenda, emphasizing on the potential to contribute meaningfully to game development by incorporating novel gaze experiences based on what HCI has considered limitations. For instance, overcoming the Midas Touch problem with calibration-free gaze interaction [77], or playing with privacy concerns of gazemonitoring to embed the player's attention in the game [76].

We are not arguing on the validity of looking at gaze from a technical perspective, as it has patently contributed to the emergence of novel and playful gaze mechanics, but we take a stand and suggest that other dimensions of gaze gameplay design should be discussed, and probably considered when creating gaze-based interaction in games.

Briefly, we want to summarize the themes discussed:

- Theme 1: How can we balance tension in the interaction to create novel game experiences? To what extent is tension playful or inhibits attention to the game tasks?

- Theme 2: How can designers leverage peripheral vision to augment gaze interaction paradigms in gameplay? What new opportunities can offer to gaze-enabled games by designing gaze interaction with peripheral vision in mind?

- Theme 3: Playing with tension, gaze side effects and antiintuitiveness as a design space in gaze-enabled gameplay.

- Theme 4: What is the influence of the "Meaning" of the gaze mechanic in gaze-based interaction? What other metaphors can be used to inspire, contextualize or augment gaze interaction in future designs? Can designers create new game opportunities by using metaphors of gaze and sight?

- Theme 5: Who is Gaze's Identity? What can gaze interaction bring to player's identity conversations? To what extent modeling Gaze's Identity and attention can create new paradigms with gaze interaction?

And lastly, could this be the new agenda for gaze interaction research in games?

\section{CONCLUSION}

This paper offers a different perspective to look at gaze interaction in games design. In doing so, we contribute to starting a conversation, and we hope to inspire new ones. Overall, the presented work is a reflection on current gaze mechanics in game design practice. We suggest topics that need to be discussed within the community and describe the opportunities thinking outside the box of eye-tracking's technical limitations could contribute to gaze interaction design.

\section{REFERENCES}

[1] Mahdieh Abbaszadegan, Sohrab Yaghoubi, and I Scott MacKenzie. 2018. TrackMaze: A Comparison of Head-Tracking, Eye-Tracking, and Tilt as Input Methods for Mobile Games. In International Conference on Human-Computer Interaction. Springer, 393-405.

[2] Saskia Bakker and Karin Niemantsverdriet. 2016. The interaction-attention continuum: considering various levels of human attention in interaction design. International Journal of Design 10, 2 (2016).

[3] Paulo Bala, Lucilia Noóbrega, Guilherme Neves, Lai’s Lopes, Joana Morna, João Camacho, and Cristina Freitas. 2015. Keyewai: Looking at Cooperation in a Holographic Projection Screen. In Proceedings of the 33rd Annual ACM Conference Extended Abstracts on Human Factors in Computing Systems. ACM, 61-64.

[4] Joseph Baldwin, Alistair Burleigh, Robert Pepperell, and Nicole Ruta. 2016. The Perceived Size and Shape of Objects in Peripheral Vision. i-Perception 7, 4 (2016), 2041669516661900.

[5] Richard Bates, Stephen Vickers, and Howell O Istance. 2010. Gaze interaction with virtual on-line communities: levelling the playing field for disabled users. Universal Access in the Information Society 9, 3 (2010), 261-272.

[6] Eric PS Baumer, Vera Khovanskaya, Mark Matthews, Lindsay Reynolds, Victoria Schwanda Sosik, and Geri Gay. 2014. Reviewing reflection: on the use of reflection in interactive system design. In Proceedings of the 2014 conference on Designing interactive systems. ACM, 93-102.

[7] Max V Birk, Cheralyn Atkins, Jason T Bowey, and Regan L Mandryk. 2016. Fostering intrinsic motivation through avatar identification in digital games. In Proceedings of the 2016 CHI Conference on Human Factors in Computing Systems. ACM, 2982-2995.

[8] David Boud, Rosemary Keogh, and David Walker. 2013. Reflection: Turning experience into learning. Routledge.

[9] Marcus Carter, Fraser Allison, John Downs, and Martin Gibbs. 2015. Player identity dissonance and voice interaction in games. In Proceedings of the 2015 Annual Symposium on Computer-Human Interaction in Play. ACM, 265-269.

[10] Marcus Carter, Martin Gibbs, and Michael Arnold. 2012. Avatars, characters, players and users: multiple identities at/in play. In Proceedings of the 24th Australian Computer-Human Interaction Conference. ACM, 68-71. 
[11] Marcus Carter, Joshua Newn, Eduardo Velloso, and Frank Vetere. 2015. Remote gaze and gesture tracking on the microsoft kinect: Investigating the role of feedback. In Proceedings of the Annual Meeting of the Australian Special Interest Group for Computer Human Interaction. ACM, 167-176.

[12] Emiliano Castellina and Fulvio Corno. 2008. Multimodal gaze interaction in 3D virtual environments. COGAIN 8 (2008), 33-37.

[13] Matthieu Perreira Da Silva, Vincent Courboulay, and Armelle Prigent. 2007. Gameplay experience based on a gaze tracking system. In "Gaze-based Creativity, Interacting with Games and On-line Communities" INPROCEEDINGS in proceedings of COGAIN 2007 (Communication by Gaze Interaction IST FP6 European Project). 25-28.

[14] Frederik De Grove, Cédric Courtois, and Jan Van Looy. 2015. How to be a gamer! Exploring personal and social indicators of gamer identity. Journal of Computer-Mediated Communication 20, 3 (2015), 346-361.

[15] Soussan Djamasbi and Siavash Mortazavi. 2015. Generation Y, baby boomers, and gaze interaction experience in gaming. In 2015 48th Hawaii International Conference on System Sciences. IEEE, 482-490.

[16] Michael Dorr, Laura Pomarjanschi, and Erhardt Barth. 2009. Gaze beats mouse: A case study on a gaze-controlled breakout. PsychNology Journal 7, 2 (2009), 197-211.

[17] Nicolas Ducheneaut, Ming-Hui Wen, Nicholas Yee, and Greg Wadley. 2009. Body and mind: a study of avatar personalization in three virtual worlds. In Proceedings of the SIGCHI conference on human factors in computing systems. ACM, 1151-1160.

[18] Inger M Ekman, Antti W Poikola, and Meeri K Mäkäräinen. 2008. Invisible eni: using gaze and pupil size to control a game. In CHI'08 extended abstracts on Human factors in computing systems. ACM, 3135-3140.

[19] Massive Entertainment. 2019. Tom Clancy's The Division 2. Game. (15 March 2019).

[20] Mary Flanagan, Daniel C Howe, and Helen Nissenbaum. 2005. Values at play: Design tradeoffs in socially-oriented game design. In Proceedings of the SIGCHI conference on human factors in computing systems. ACM, 751-760.

[21] Rowanne Fleck and Geraldine Fitzpatrick. 2010. Reflecting on reflection: framing a design landscape. In Proceedings of the 22nd Conference of the Computer-Human Interaction Special Interest Group of Australia on Computer-Human Interaction. ACM, 216-223.

[22] Tobii Gaming. PC Games with Eye Tracking gameplay. (????). https://gaming. tobii. com/games
[23] William W Gaver, Jacob Beaver, and Steve Benford. 2003. Ambiguity as a resource for design. In Proceedings of the SIGCHI conference on Human factors in computing systems. ACM, 233-240.

[24] James Paul Gee. 2003. What video games have to teach us about learning and literacy. Computers in Entertainment (CIE) 1, 1 (2003), 20-20.

[25] Casper Harteveld, Rui Guimarães, Igor S Mayer, and Rafael Bidarra. 2010. Balancing play, meaning and reality: The design philosophy of LEVEE PATROLLER. Simulation \& Gaming 41, 3 (2010), 316-340.

[26] Sébastien Hillaire, Anatole Lécuyer, Rémi Cozot, and Géry Casiez. 2008. Using an eye-tracking system to improve camera motions and depth-of-field blur effects in virtual environments. In Virtual Reality Conference, 2008. VR'08. IEEE. IEEE, 47-50.

[27] Zaheer Hussain and Mark D Griffiths. 2008. Gender swapping and socializing in cyberspace: An exploratory study. CyberPsychology \& Behavior 11, 1 (2008), 47-53.

[28] Beyond Infinity. 2015. Glimpse of Fear. Game. (22 October 2015).

[29] Katherine Isbister, Mary Flanagan, and Chelsea Hash. 2010. Designing games for learning: insights from conversations with designers. In Proceedings of the SIGCHI Conference on Human Factors in Computing Systems. ACM, 2041-2044.

[30] Poika Isokoski, Markus Joos, Oleg Spakov, and Benoît Martin. 2009. Gaze controlled games. Universal Access in the Information Society 8, 4 (2009), 323-337.

[31] Poika Isokoski and Benot Martin. 2006. Eye tracker input in first person shooter games. In Proceedings of the 2nd Conference on Communication by Gaze Interaction: Communication by Gaze Interaction-COGAIN 2006: Gazing into the Future. 78-81.

[32] Howell Istance, Aulikki Hyrskykari, Lauri Immonen, Santtu Mansikkamaa, and Stephen Vickers. 2010. Designing gaze gestures for gaming: an investigation of performance. In Proceedings of the 2010 Symposium on Eye-Tracking Research \& Applications. ACM, 323-330.

[33] Howell Istance, Aulikki Hyrskykari, Stephen Vickers, and Thiago Chaves. 2009. For your eyes only: Controlling $3 \mathrm{~d}$ online games by eye-gaze. In Human-Computer Interaction-INTERACT 2009. Springer, 314-327.

[34] Robert J. K. Jacob. 1990. What you look at is what you get: eye movement-based interaction techniques. In Proceedings of the SIGCHI conference on Human factors in computing systems: Empowering people (CHI '90). ACM, New York, NY, USA, 11-18. DOI : http://dx.doi .org/10.1145/97243.97246 
[35] Shahram Jalaliniya and Diako Mardanbegi. 2016. Eyegrip: Detecting targets in a series of uni-directional moving objects using optokinetic nystagmus eye movements. In Proceedings of the 2016 CHI Conference on Human Factors in Computing Systems. ACM, 5801-5811.

[36] Rilla Khaled. 2018. Questions over answers: Reflective game design. In Playful Disruption of Digital Media. Springer, 3-27.

[37] Mohamed Khamis, Carl Oechsner, Florian Alt, and Andreas Bulling. 2018. VRpursuits: interaction in virtual reality using smooth pursuit eye movements. (2018).

[38] Michael Lankes, Thomas Mirlacher, Stefan Wagner, and Wolfgang Hochleitner. 2014. Whom are you looking for?: the effects of different player representation relations on the presence in gaze-based games. In Proceedings of the first ACM SIGCHI annual symposium on Computer-human interaction in play. ACM, 171-179.

[39] Michael Lankes and Barbara Stiglbauer. 2016. GazeAR: Mobile gaze-based interaction in the context of augmented reality games. In International Conference on Augmented Reality, Virtual Reality and Computer Graphics. Springer, 397-406.

[40] Michael Lankes and Stefan Wagner. 2015. Taking a Look at the Player's Gaze: The Effects of Gaze Visualizations on the Perceived Presence in Games.. In DiGRA Conference.

[41] Bernhard Maurer, Ilhan Aslan, Martin Wuchse, Katja Neureiter, and Manfred Tscheligi. 2015. Gaze-based onlooker integration: exploring the in-between of active player and passive spectator in co-located gaming. In Proceedings of the 2015 Annual Symposium on Computer-Human Interaction in Play. ACM, 163-173.

[42] Elisa D Mekler, Ioanna Iacovides, and Julia Ayumi Bopp. 2018. A Game that Makes You Question...: Exploring the Role of Reflection for the Player Experience. In Proceedings of the 2018 Annual Symposium on Computer-Human Interaction in Play. ACM, 315-327.

[43] Eidos Montreal. 2018. Shadow of the Tomb Raider. Game. (14 September 2018).

[44] Sheila C Murphy. 2004. 'Live in your world, play in ours': The spaces of video game identity. Journal of visual culture 3, 2 (2004), 223-238.

[45] Lennart Erik Nacke, Michael Kalyn, Calvin Lough, and Regan Lee Mandryk. 2011. Biofeedback game design: using direct and indirect physiological control to enhance game interaction. In Proceedings of the SIGCHI conference on human factors in computing systems. ACM, 103-112.

[46] Lennart E Nacke, Sophie Stellmach, Dennis Sasse, and Craig A Lindley. 2010. Gameplay experience in a gaze interaction game. arXiv preprint arXiv:1004.0259 (2010).
[47] Dong Nguyen. 2013. Flappy Bird. Game [Phone]. (24 May 2013).

[48] Anders Møller Nielsen, Anders Lerchedahl Petersen, and John Paulin Hansen. 2012. Gaming with gaze and losing with a smile. In Proceedings of the Symposium on Eye Tracking Research and Applications. ACM, 365-368.

[49] J O'Donovan, J Ward, S Hodgins, and V Sundstedt. 2009. Rabbit run: Gaze and voice based game interaction. In Eurographics Ireland Workshop, December.

[50] Pablo Ortiz and D Fox Harrell. 2018. Enabling Critical Self-Reflection through Roleplay with Chimeria: Grayscale. In Proceedings of the 2018 Annual Symposium on Computer-Human Interaction in Play. ACM, 353-364.

[51] Ken Pfeuffer, Jason Alexander, and Hans Gellersen. 2016. GazeArchers: Playing with Individual and Shared Attention in a Two-player Look\&Shoot Tabletop Game. In Proceedings of the 15th International Conference on Mobile and Ubiquitous Multimedia (MUM'16). ACM, New York, NY, USA, 213-216. DOI: http://dx.doi.org/10.1145/3012709.3012717

[52] Argenis Ramirez Gomez and Hans Gellersen. 2019. SuperVision: Playing with Gaze Aversion and Peripheral Vision. In Proceedings of the 2019 CHI Conference on Human Factors in Computing Systems. ACM, 473.

[53] Ruth Rosenholtz. 2016. Capabilities and limitations of peripheral vision. Annual Review of Vision Science 2 (2016), 437-457.

[54] Pedro Sanches, Kristina Hook, Corina Sas, and Anna Stahl. 2019. Ambiguity as a resource to inform proto-practices: The case of skin conductance. $A C M$ Transactions on Computer-Human Interaction (TOCHI) (2019).

[55] C Schaefer, R Menges, K Schmidt, M Kuich, and T Walber. 2014. Schau genau! an eye tracking game with a purpose. Applications for Gaze in Games (2014).

[56] Phoebe Sengers, Kirsten Boehner, Shay David, and Joseph'Jofish' Kaye. 2005. Reflective design. In Proceedings of the 4th decennial conference on Critical computing: between sense and sensibility. ACM, 49-58.

[57] J David Smith and TC Graham. 2006. Use of eye movements for video game control. In Proceedings of the 2006 ACM SIGCHI international conference on Advances in computer entertainment technology. ACM, 20.

[58] India Starker and Richard A. Bolt. 1990. A Gaze-responsive Self-disclosing Display. In Proceedings of the SIGCHI Conference on Human Factors in Computing Systems (CHI '90). ACM, New York, NY, USA, 3-10. DOI :

http://dx.doi.org/10.1145/97243.97245 
[59] Robert Louis Stevenson. 2018. Strange Case of Dr Jekyll and Mr Hyde. In Medicine and Literature, Volume Two. CRC Press, 105-118.

[60] Starbreeze Studios. 2013. Brothers: A Tale of Two Sons. Game. (7 August 2013).

[61] Stillalive Studios. 2015. Son of Nor. Game. (28 April 2015).

[62] Techland. 2018. Dying Light: Bad Blood. Game. (13 September 2018).

[63] Sabine Trepte and Leonard Reinecke. 2010. Avatar creation and video game enjoyment. Journal of Media Psychology (2010).

[64] Jayson Turner, Eduardo Velloso, Hans Gellersen, and Veronica Sundstedt. 2014. EyePlay: applications for gaze in games. In Proceedings of the first ACM SIGCHI annual symposium on Computer-human interaction in play. ACM, 465-468.

[65] Ubisoft. 2016. Watch Dogs 2. Game. (29 November 2016).

[66] Ubisoft. 2018. Assassin's Creed Odyssey. Game. (5 October 2018).

[67] Ubisoft Montreal and Ubisoft Toronto. 2018. Far Cry 5. Game [Xbox][Windows][PlayStation]. (27 March 2018).

[68] MUHTAR ÇAĞKAN ULUDAĞLI and CENGİZ ACARTÜRK. 2018. User interaction in hands-free gaming: a comparative study of gaze-voice and touchscreen interface control. Turkish Journal of Electrical Engineering \& Computer Sciences 26, 4 (2018), 1967-1976.

[69] Eva A Van Reijmersdal, Jeroen Jansz, Oscar Peters, and Guda Van Noort. 2013. Why girls go pink: Game character identification and game-players' motivations. Computers in Human Behavior 29, 6 (2013), 2640-2649.

[70] Boris Velichkovsky, Andreas Sprenger, and Pieter Unema. 1997. Towards gaze-mediated interaction: Collecting solutions of the "Midas touch problem". In Human-Computer Interaction INTERACTâĂŹ97. Springer, 509-516.

[71] Eduardo Velloso and Marcus Carter. 2016. The Emergence of EyePlay: A Survey of Eye Interaction in Games. In Proceedings of the 2016 Annual Symposium on Computer-Human Interaction in Play (CHI PLAY '16). ACM, New York, NY, USA, 171-185. DOI : http://dx.doi.org/10.1145/2967934.2968084

[72] Eduardo Velloso, Amy Fleming, Jason Alexander, and Hans Gellersen. 2015a. Gaze-Supported Gaming: MAGIC Techniques for First Person Shooters. In Proceedings of the 2015 Annual Symposium on
Computer-Human Interaction in Play (CHI PLAY'15). ACM, New York, NY, USA, 343-347. DOI : http://dx. doi .org/10.1145/2793107.2793137

[73] Eduardo Velloso, Carl Oechsner, Katharina Sachmann, Markus Wirth, and Hans Gellersen. 2015b. Arcade+: A Platform for Public Deployment and Evaluation of Multi-Modal Games. In Proceedings of the 2015 Annual Symposium on Computer-Human Interaction in Play (CHI PLAY '15). ACM, New York, NY, USA, 271-275. DOI : http://dx. doi .org/10.1145/2793107.2793145

[74] Stephen Vickers, Howell Istance, and Matthew Smalley. 2010. EyeGuitar: making rhythm based music video games accessible using only eye movements. In Proceedings of the 7th International Conference on Advances in Computer Entertainment Technology. ACM, 36-39.

[75] Melodie Vidal. 2014. Shynosaurs: A Game of Attention Dilemma. In Proceedings of the First ACM SIGCHI Annual Symposium on Computer-human Interaction in Play (CHI PLAY '14). ACM, New York, NY, USA, 391-394. DOI:

http://dx . doi .org/10.1145/2658537.2662979

[76] Melodie Vidal, Remi Bismuth, Andreas Bulling, and Hans Gellersen. 2015. The Royal Corgi: Exploring Social Gaze Interaction for Immersive Gameplay. In Proceedings of the 33rd Annual ACM Conference on Human Factors in Computing Systems (CHI '15). ACM, New York, NY, USA, 115-124. DOI: http://dx.doi.org/10.1145/2702123.2702163

[77] Mélodie Vidal, Andreas Bulling, and Hans Gellersen. 2013. Pursuits: Spontaneous Interaction with Displays Based on Smooth Pursuit Eye Movement and Moving Targets. In Proceedings of the 2013 ACM International Joint Conference on Pervasive and Ubiquitous Computing (UbiComp '13). ACM, New York, NY, USA, 439-448. DOI :

http://dx.doi .org/10.1145/2493432.2493477

[78] David Whitney and Dennis M Levi. 2011. Visual crowding: A fundamental limit on conscious perception and object recognition. Trends in cognitive sciences 15 , 4 (2011), 160-168.

[79] Tom Wilcox, Mike Evans, Chris Pearce, Nick Pollard, and Veronica Sundstedt. 2008. Gaze and voice based game interaction: the revenge of the killer penguins. SIGGRAPH Posters 81 (2008).

[80] Oscar Wilde and Joseph Bristow. 2006. The picture of dorian gray. OUP Oxford.

[81] Shumin Zhai, Carlos Morimoto, and Steven Ihde. 1999. Manual and gaze input cascaded (MAGIC) pointing. In Proceedings of the SIGCHI conference on Human Factors in Computing Systems. ACM, 246-253. 